\title{
Design of multi-range tomographic system for transport studies in tokamak plasmas
}

\author{
V. Weinzettl ${ }^{\mathrm{a},{ }^{*}}$, D. I. Naydenkova a , D. Sestak ${ }^{\mathrm{a}}$, J. Vlcek ${ }^{\mathrm{a}}$, J. Mlynar ${ }^{\mathrm{a}}$, R. Melich ${ }^{\mathrm{a}}$, \\ D. Jares ${ }^{\mathrm{a}}$, J. Malot ${ }^{\mathrm{b}}$, D. Sarychev ${ }^{\mathrm{c}}$, V. Igochine ${ }^{\mathrm{d}}$ \\ ${ }^{a}$ Institute of Plasma Physics, Association EURATOM/IPP.CR, Prague, Czech Republic \\ ${ }^{b}$ Arts et Metiers ParisTech, Paris, France \\ 'NFI RRC "Kurchatov Institute", Moscow, Russia \\ ${ }^{d}$ Max-Planck-Institut für Plasmaphysik, EURATOM Association, Garching, Germany
}

Elsevier use only: Received date here; revised date here; accepted date here

\begin{abstract}
The integrated spectroscopic system for visible plasma radiation, soft X-ray, and bolometric measurements has been designed for the COMPASS tokamak. This diagnostic allows tomographic reconstruction at a few microseconds time base and features an improved spatial resolution about one centimeter in the pedestal region, where the highest gradient of plasma pressure drives turbulent structures and their propagation towards the wall. In combination with other developed diagnostics on COMPASS, it represents an ideal tool for a measurement and characterization of these radiating plasma structures which are responsible for an extremely high particle/energy transport across magnetic field. The design of the new integrated multichannel spectroscopic diagnostics is reviewed and corresponding technical challenges are described. Additionally, the methods, which will be used for data processing, are briefly mentioned and target physics is discussed. (C) 2001 Elsevier Science. All rights reserved
\end{abstract}

Keywords: SXR; bolometry; visible light; tokamak; COMPASS

\section{Introduction}

The COMPASS tokamak, a divertor device with clear H-mode and ITER-relevant geometry (1:10 to ITER plasma size, $\mathrm{R}=0.56 \mathrm{~m}, \mathrm{a}=0.23 \times 0.38 \mathrm{~m}$, $\mathrm{Ip}<400 \mathrm{kA}, \mathrm{BT}=1.2-2.1 \mathrm{~T}$ and pulse length up to $1 \mathrm{~s}$ ), has been re-installed at IPP Prague after its transport from Culham in UK [1]. Many new diagnostic tools with both high temporal and spatial resolutions are under development to address the scientific program focused on H-mode physics and pedestal investigations.

In the second section of the article, a physical motivation for a construction of the integrated multichannel spectroscopic system for visible plasma radiation, soft X-ray, and bolometric measurements is introduced. In the third part, the final design of this complex system for the COMPASS tokamak is

\footnotetext{
* Corresponding author. Tel.: +420-266052947; fax: +420-286586389; e-mail: vwei@ipp.cas.cz.
} 
described. In the last section, possible data processing methods are mentioned.

\section{Physical motivation}

The problem of anomalous particle/energy transport in fusion research oriented to magnetically confined plasma has been studied for many decades. In recent years, fast progress in development of different spectroscopic tools with high temporal and/or spatial resolution has occurred. Parameters improvement of the applied detection systems and major advances in computer simulations permit to make comparative studies between complex theoretical models and new experimental data. However, basic questions concerning mechanisms of anomalous transport like the creation and behavior of turbulent structures [3], the role of plasma impurities [4], the influence of plasma-wall interaction on edge plasma parameters [5], and consequently on the particle confinement and tokamak performance [6] still remain open. Current experiments in many devices indicate a possibility to measure turbulent filaments in visible light emission corresponding mainly to density fluctuations at the edge [7]. Other facilities [8] validated possibility to measure these plasma structures and very fast events using AXUVbased bolometric detectors. A connection between the edge activity and the core behavior was demonstrated in [9] in the data analysis from fast SXR and AXUV detectors. Therefore, the integrated multi-channel spectroscopic system for fast visible plasma radiation, soft $\mathrm{X}$-ray, and bolometric measurements was proposed for a construction on the COMPASS tokamak. This system will be further supported by other toroidally separated diagnostics with high temporal and/or spatial resolution like fast cameras, high-resolution Thomson scattering, reflectometry and advanced probe types. It will represent an ideal tool for measurement and characterization of the above-mentioned radiating plasma structures responsible for an extremely high particle/energy transport across magnetic field in tokamaks.

\section{Design of multi-range tomographic system for the COMPASS tokamak}

New multi-channel spectroscopic diagnostic port plugs containing the systems for visible plasma radiation, soft X-ray (SXR), and bolometric measurements were designed so that acceptable viewing angles are achieved in the significantly constrained space available in the only set of diagnostic ports of the COMPASS tokamak (sector 6/7) that allows for the future extended configuration planned for tomographic applications, see Fig.1.

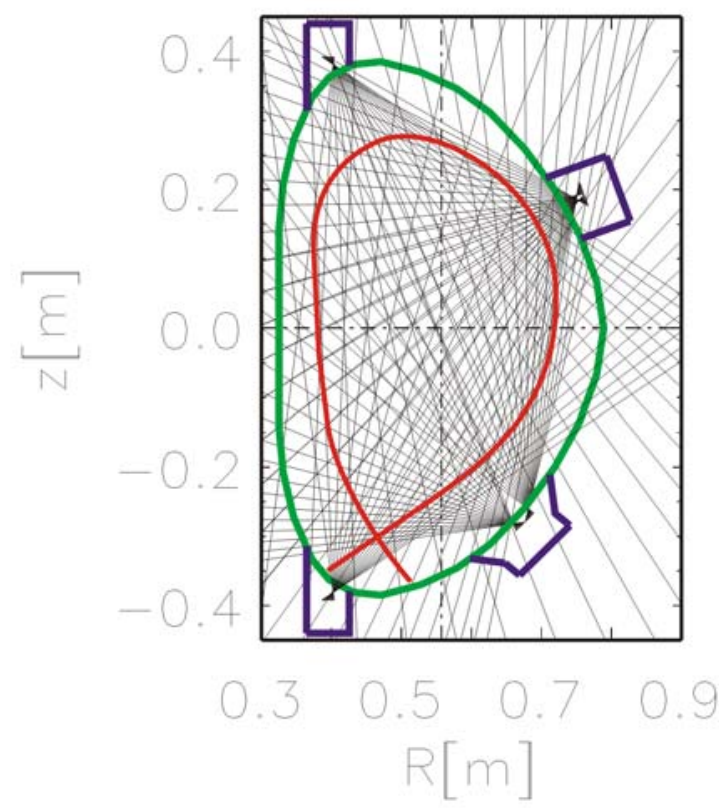

Fig. 1. The set of diagnostic ports for tomography and the coverage of the poloidal cross section by bolometric chords.

There are two angular ports of the NW100 type and two low-accessible vertical ports of the NW63 type. First, a distribution of the detection systems and suitable detector types for both fast bolometry and SXR observations and a concept for optical path of the visible light had to be chosen. The port dimensions and the fact that it is locked deep between magnetic field coils limited the design in the following way: The angular plugs can contain two arrays of twenty channel AXUV-based silicon bolometers AXUV20EL from IRD, a thirty-five channel SXR silicon sensor LD35-5T from Centronic 
and a special custom-made ultra wide-angle lensbased objective for a multi-channel visible light diagnostic (VIS), developed in IPP Prague. The vertical ports are even smaller; therefore, they can accommodate only one twenty channel bolometric or SXR detector. Furthermore, the port plugs must be equipped with shutters to prevent detectors and optics from deposition of impurity layers during a cleaning glow discharge, and with a water cooling channel in order to protect the detectors from overheating during a vacuum vessel baking.

The plug for the angular ports is assembled from two pieces in a "sandwich" arrangement, see Fig.2. The first one holds detectors, air slits, shutter,

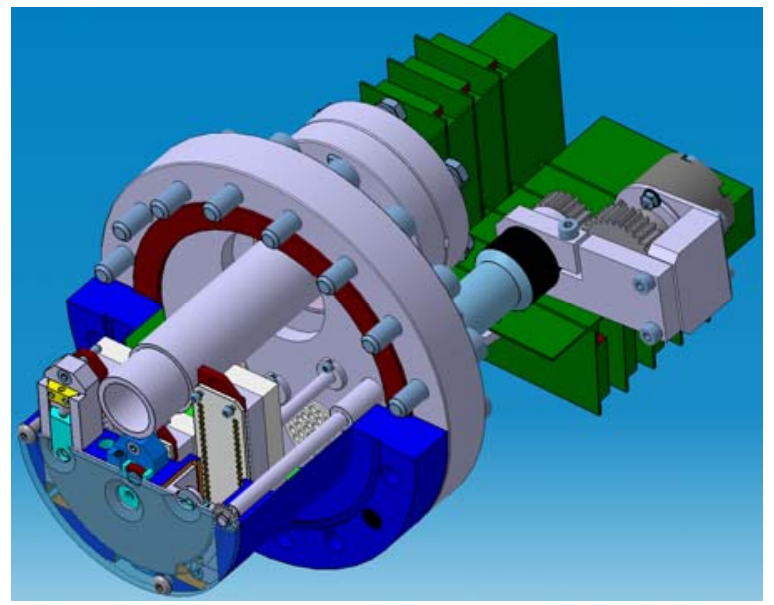

Fig. 2. The angular port plug design. The left part integrates the detectors, air slits, shutter, optics and cooling channel. The middle part contains two electrical feedthroughs, the NW16 flange for the shutter manipulator and another opening for an NW35 viewport. On the right, the shutter driving mechanism and amplifiers are located.

and also includes a cooling channel. It was designed as one monolithic compact inset. The cooling channel is drilled through the inset; the openings are sealed by threaded stoppers and welded on the vacuum side. The plasma-facing bolometric and SXR detectors are mounted on the ceramic sockets and are covered by an air slit holder. The air slit holder is used for positioning of the slit with respect to the detector center, when a change of the observation angle is required. As a collimating element the $0.1 \mathrm{~mm}$ wide and $2 \mathrm{~mm}$ long air slit together with the second similar slit in perpendicular direction can be used. The air slits are replaceable allowing for an independent change of the vertical and toroidal spatial resolutions. Increasing the toroidal view, mainly the radiation throughput is influenced due to toroidal symmetry of the observed plasma. In the case of soft X-rays, a thin beryllium foil of $5 \mu \mathrm{m}$ is used to set the lower energy threshold to several hundreds $\mathrm{eV}$, i.e. to cut VIS and UV radiation. The second piece of the plug is a NW100 flange that contains two electrical feedthroughs, each equipped with 41 pins, one opening for a rotary feedthrough, a NW16 flange - intended for the shutter manipulator and another opening for a NW35 viewport for VIS observations. The NW35 vacuum window is made of Spectrosil 2000 by Vacom that is transparent for a wide wavelength range around visible light. The visible light diagnostic system relies on a light transmitted through the focusing lens-based objective located inside the inset and the NW100 flange. The objective consists of three parts that are optimized as a unit and compensate their optical aberration. The first part, an ultra wide-angle objective achieving $114^{\circ}$ field of view, transmits an image of one half of the plasma column into infinity. The second part is an infinite-finite distance objective that creates a real image. The third part is a system of relay lens reimaging it onto a linear set of optical fibers directly or through an interference filter. In this part, the throughput is restricted by a maximum possible diameter of the viewport and set-up of the fiber bundle that strongly depends on the available budget. In the presented case, only one silica/silica fiber of $200 \mu \mathrm{m}$ core represents each spatial channel. High flexibility of the fiber set-up is reached using exchangeable endpieces that allow for fiber reconnection penalised by a few per cent loss of light. Twenty meter long optical cables are led - depending on purpose of the measurement - either to a 35channel detector S4114-35Q by Hamamatsu, or to minispectrometers HR2000+ by Ocean Optics with spectral coverage 248-472 nm and 457-663 nm, with approximate spectral resolution $0.15 \mathrm{~nm}$. All the three detection systems are equipped with an integrated shutter controlled via the NW35 rotary feedthrough.

The space around the vertical ports is narrower preventing to use the same "sandwich" configuration as in the angular ports. The port plug is made of the cap and two welded parts that allows the 
cooling pipes and the electrical and mechanical feedthrough to be positioned on the same piece. The cooling system is drilled through the inner part of the flange and finished by a cap which is welded and manufactured to sustain 6 bars pressure of the demineralized water. Space limitations led to optimizations also in cabling, where an interconnector instead of individual cables will be fixed to the second part of the port plug in order to allow for a simple connection at once. The air slit holder was improved in order to achieve a better performance, i.e. an easier movement of the slit in the direction parallel to the detector surface plus a new possibility of its vertical shifting via changes of the slit holder. Again, a shutter system controlled by a pneumatic engine allows for protection of the sensor during a glow discharge.

All the three detection systems provide line integrated measurements along spatially stepped lines of sight with resolution about 1-2 cm depending on a set of viewing angles. Temporal resolution of all systems based on silicon arrays is given by an integration constant of the amplifier that is limited approximately to $1 \mathrm{MHz}$. Data acquisitions based on the ATCA set-up [10] use $2 \mathrm{MHz}$ sampling rate. The minispectrometers are capable for recording spectra with up to $1 \mathrm{kHz}$ temporal resolution.

\section{Data processing methods}

In order to extract fast processes and their localization, a method based on Singular Value Decomposition will be applied independently [11-12] or as a data filter for an input to tomography techniques [13]. In previous years, a new robust algorithm for fast tomography was developed and tested on TCV [14] and implemented also at JET. This algorithm - based on Tikhonov regularization with iterative minimizing of Fisher information in the reconstructed image and instantaneous processing of multiple timeframes - is foreseen to be applied at COMPASS, with significant potential for real-time applications after minor modification. Correlation analysis applied on fast bolometric signals [15] presents another high-performance tool visualizing repetitive processes.

\section{Conclusion}

The unique integrated spectroscopic system allowing for tomographic reconstruction at a few microseconds time base with spatial resolution of about one centimeter was introduced. In combination with other developed diagnostics on the COMPASS tokamak it will serve for a characterization of plasma structures responsible for anomalous transport.

\section{Acknowledgments}

The work was performed and supported from the grant GA CR No. 202/09/1467, and the engineering part by the EFTS project EODI No. 042884.

\section{References}

[1] R. Panek, et al., Czech. J. Phys. 56 (2006) B125-137

[2] D. Sestak, et al., Fusion Eng. Des. 84 (2009) 1755-58

[3] V. Budaev, et al., Nucl. Fusion 44 (2004) S108-117

[4] L. Krlin, et al., Czech. J. Phys. 54 (2004) C157-163

[5] U. Samm, Plasma Phys. Control. Fusion 41 (1999) B57-76

[6] T. Fukuda, Plasma Phys. Control. Fusion 40 (1998) 543-555

[7] R. Maqueda, et al., Rev. Sci. Instrum., Vol. 72, No. 1, January 2001, p. 931-4

[8] A. Degeling et al., "The AXUV Bolometer and Lyman-a Emission Camera Systems on the TCV Tokamak”, $15^{\text {th }}$ Topical Conf. on High-Temperature Plasma Diagnostics, 1922 April 2004, San Diego, USA

[9] Y.R. Martin, A.W. Degeling and J.B. Lister, Plasma Phys. Control. Fusion 44 (2002) A373-382

[10] D.F. Valcárcel, et al., Fus. Eng. Des., Volume 84 (2009), Issue 7-11, pp. 1901-1904

[11] V.Weinzettl, et al., $33^{\text {rd }}$ EPS Conference on Plasma Physics, Roma, Italy, ECA Vol. 30I (2006) P-4.080

[12] G.Van Oost, et al., Nuclear Fusion 47 (2007) 378-386

[13] J. Mlynar, et al., Fus. Eng. Des. 66-68 (2003) 821

[14] J. Mlynar, et al., Plasma Phys. Control. Fusion 45 (2003) 169180

[15] M. Gryaznevich, et al., Nucl. Fusion 49 (2009) 10402 